\title{
A novel method to determine the natural course of unruptured brain arteriovenous malformations without the need for follow-up information
}

\author{
Bengt Karlsson, MD, PhD, ${ }^{1}$ Arne V. Johansson, PhD, ${ }^{2}$ Huai-Che Yang, MD, ${ }^{3}$ Hidefumi Jokura, MD, ${ }^{4}$ \\ Masaaki Yamamoto, MD, ${ }^{5}$ Roberto Martínez-Álvarez, MD, ${ }^{6}$ Jun Kawagishi, MD, ${ }^{4}$ \\ Wan-Yuo Guo, MD, PhD, ${ }^{9}$ Guus Beute, MD, ${ }^{7}$ David H. C. Pan, MD, ${ }^{3}$ Wen-Yuh Chung, MD, ${ }^{3}$ \\ Michael Söderman MD, PhD, ${ }^{8}$ Hitoshi Aiyama, MD, ${ }^{5}$ and Tseng Tsai Yeo, MBBS, FRACS ${ }^{1}$
}

\begin{abstract}
'Division of Neurosurgery, Department of Surgery, National University Hospital, Singapore; '2Department of Mechanics, KTH Royal Institute of Technology, Stockholm, Sweden; Departments of ${ }^{3}$ Neurosurgery and ${ }^{~}$ Radiology, Veterans General Hospital, Taipei, Taiwan; ${ }^{4}$ Jiro Suzuki Memorial Gamma House, Furukawa Seiryo Hospital, Osaki, Japan; ${ }^{5}$ Katsuta Hospital Mito GammaHouse, Ibaraki, Japan; ${ }^{6}$ Ruber International Hospital, Madrid, Spain; ${ }^{7} E T Z$ Elizabeth, Tilburg, The Netherlands; and ${ }^{8}$ Karolinska Hospital, Stockholm, Sweden
\end{abstract}

\begin{abstract}
OBJECTIVE There is a strong clinical need to accurately determine the average annual hemorrhage risk in unruptured brain arteriovenous malformations (AVMs). This need motivated the present initiative to use data from a uniquely large patient population and design a novel methodology to achieve a risk determination with unprecedented accuracy. The authors also aimed to determine the impact of sex, pregnancy, AVM volume, and location on the risk for AVM rupture.

METHODS The present study does not consider any specific management of the AVMs, but only uses the age distribution for the first hemorrhage, the shape of which becomes universal for a sufficiently large set of patients. For this purpose, the authors collected observations, including age at first hemorrhage and AVM size and location, in 3425 patients. The average annual risk for hemorrhage could then be determined from the simple relation that the number of patients with their first hemorrhage at a specific age equals the risk for hemorrhage times the number of patients at risk at that age. For a subset of the patients, the information regarding occurrence of AVM hemorrhage after treatment of the first hemorrhage was used for further analysis of the influence on risk from AVM location and pregnancy.
\end{abstract}

RESULTS The age distribution for the first AVM hemorrhage was used to determine the average annual risk for hemorrhage in unruptured AVMs at adult ages (25-60 years). It was concluded to be $3.1 \% \pm 0.2 \%$ and unrelated to AVM volume but influenced by its location, with the highest risk for centrally located AVMs. The hemorrhage risk was found to be significantly higher for females in their fertile years.

CONCLUSIONS The present methodology allowed the authors to determine the average annual risk for the first AVM hemorrhage at $3.1 \% \pm 0.2 \%$ without the need for individual patient follow-up. This methodology has potential also for other similar types of investigations. The conclusion that centrally located AVMs carry a higher risk was confirmed by follow-up information. Follow-up information was also used to conclude that pregnancy causes a substantially greater AVM hemorrhage risk. The age distribution for AVM hemorrhage is incompatible with AVMs present at birth having the same hemorrhage risk as AVMs in adults. Plausibly, they instead develop in the early years of life, possibly with a lower hemorrhage risk during that time period.

https://thejns.org/doi/abs/10.3171/2018.7.GKS181278

KEYWORDS AVM; hemorrhage; risk; vascular disorders; stereotactic radiosurgery 
$\mathrm{M}$ ANY studies estimating the annual risk for hemorrhage of unruptured arteriovenous malformations (AVMs) have been published. . $^{2,4,5,8,10-13,15-17,}$ 20,23,25 With few exceptions, the studies are retrospective and analyze patients in whom, for various reasons, the AVM has been left untreated for longer or shorter periods of time. In most reports, the risk of rupture varies between $2 \%$ and $4 \%$, but a rate exceeding $30 \%$ was reported for select ruptured AVMs. ${ }^{20,31}$ Several factors have been suggested to influence the risk for hemorrhage, such as AVM volume and location, angioarchitecture, history of earlier rupture, drainage pattern, venous stenosis, aneurysm, pregnancy, age, sex, and observation time.

More recently, the hypothesis that previously unruptured AVMs carry a lower risk for rupture than those that have hemorrhaged has been highlighted. ${ }^{17,23}$ This led to the ARUBA (A Randomized Trial of Unruptured Brain Arteriovenous Malformations) study, concluding that the annual hemorrhage risk for unruptured AVMs is, within a likelihood of $95 \%$, between $0.9 \%$ and $4.5 \%$, with an average value given as $2.2 \%$. The ARUBA study highlighted the need for a more accurate estimate of the risk for hemorrhage in AVMs to enable proper counseling of AVM patients. ${ }^{23}$

The variation in the estimation of the annual risk for AVM rupture suggests that the methodology used is suboptimal. As the incidence of hemorrhages is low, a large patient population is required to be monitored for many years to result in accurate risk estimation. Fatal hemorrhages in patients with earlier undiagnosed AVMs are likely to be underreported. The observation that the risk for hemorrhage decreases with time, also for untreated and unruptured AVMs, is more likely explained by methodological study errors than by changes in the AVMs.

In 1997, we introduced a novel method to define the risk for AVM hemorrhage, hereafter referred to as KLJS. ${ }^{15}$ The basis of this method was to use the age distribution for the first AVM hemorrhage in a large patient population. The major advantage with this method is that follow-up data are not needed. AVMs were assumed to be congenital, which was the prevailing opinion at that time. The primary conclusion was that the risk for hemorrhage increases with age; for example, that the annual risk for rupture is $6 \%$ for a 40 -year-old and $8 \%$ for a 50 -year-old individual. This result is not compatible with our clinical experience.

As a consequence, we need to challenge the opinion that AVMs are congenital, as Lasjaunias did in 1997.18 Petridis et al. analyzed data from all patients admitted in Germany during the years 2009-2013 and concluded that the data contradict the assumption that AVMs are congenital. ${ }^{28}$ The fact that de novo AVMs not infrequently appear after complete removal or obliteration of AVMs in children shows that at least some AVMs develop in the earlier years of life. ${ }^{1,9,14,19,26}$ The diagnosis of intrauterine AVMs is extremely rare, and $50 \%-80 \%$ of them present with hemorrhage, ${ }^{6}$ which contradicts the prerequisites of AVMs being congenital and initially benign to be compatible with the conclusions in the KLJS study.

The logical explanation to this is that AVMs are developmental rather than congenital and develop as long as the brain develops, i.e., until the age of around 25 years. We thus decided to perform a study hypothesizing that it is possible to estimate the annual risk for AVM hemorrhage in earlier nonruptured AVMs by using the same type of methodology in a larger number of patients and discarding the assumption that AVMs are congenital.

\section{Methods}

The concept in this study is that it is possible to assess the risk for the initial hemorrhage in nonruptured AVMs by analyzing the age distribution of the first hemorrhage. Note that the present study does not consider any specific treatment of the AVMs, but only uses the data of the patient's age at first hemorrhage. The assumption that AVMs are congenital was discarded in our analysis. The first part of the study was to show that the age distribution for the first AVM hemorrhage is universal when normalized by the total number of patients observed, if the patient population is large enough. We assumed as a first approximation that the average risk is age independent. The study design enabled us to exclude the potential flaws with possible selection bias and incomplete follow-up data inherent with conventional studies.

The study was based on "old" data from the 1479 patients included in the KLJS study ${ }^{15}$ and "new" data from the following hospitals participating in the study: Taipei Veterans General Hospital; Jiro Suzuki Memorial Gamma House, Furukawa Seiryo Hospital; Ruber International Hospital, Madrid; Katsuta Hospital Mito GammaHouse, Ibaraki; ETZ Elizabeth, Tilburg; and National University Hospital, Singapore. IRB approval was obtained from each of the participating institutions. In addition, data from one of the authors' personal series (B.K.) was added to the "new" data. The data were anonymized before being added to the study. One of the authors (B.K.) visited all institutions to ensure the accuracy of the collected data.

Data regarding patient sex and the age at the first hemorrhage were collected for 1803 male and 1622 female patients, giving a total of 3425 patients. All patients experienced their hemorrhage before any of the AVMs were treated. All patients had imaging evidence of a hemorrhage and were referred to a neurosurgical clinic. Information about the AVM location was available for 3311 patients, being central in 1258 , cerebellar in 345 , and peripheral in 1708 patients. A central location was defined as an AVM located in the brainstem, thalamus, basal ganglia, or intraor periventricular region. All other supratentorial lesions were defined as peripheral. The AVM volume, defined as the AVM nidus only, thus excluding the feeders and draining veins, was known in 2880 patients, with 1632 AVMs $\leq$ $2 \mathrm{~cm}^{3}$ and $312 \geq 10 \mathrm{~cm}^{3}$. The first treatment for the AVM was Gamma Knife surgery (GKS) in 2322 patients, while 1103 were initially managed with other treatment modalities, including no treatment.

\section{Annual Risk of Hemorrhage}

The age distribution of incidence of AVM hemorrhage in the patient material considered here is denoted as $\mathrm{n}(\mathrm{x})$, representing the number of hemorrhages per year at age $\mathrm{x}$. In the following analysis, we will use the moving 5-year 
average representation of the data. One should keep in mind that we can consider the distribution shape to be independent of the number of patients for a large enough population.

We will consider a virtual population, $\mathrm{N}(\mathrm{x})$, with a distribution over age, $\mathrm{x}$, that we seek to determine from the information contained in the age distribution of the observed AVM hemorrhages. In this procedure, we used the assumptions of an age-independent annual risk in conjunction with the above conclusion of noncongenital AVMs, implying that the population is empty at age zero, i.e., $\mathrm{N}(0)=0$. Thus, $\mathrm{N}(\mathrm{x})$ will increase up to some age (that will follow from the analysis) and thereafter be constant, except for some decrease at older ages, which can be expected from deaths due to non-AVM-related factors. At any given age, $x$, the number of observed hemorrhages, $\mathrm{n}(\mathrm{x})$, should be equal to the annual risk, $\mathrm{r}$, multiplied by the number of AVMs at risk for their first hemorrhage. The annual risk is here assumed to be independent of age. The number of AVMs at risk equals the number of AVMs developed up to year x (i.e., $\mathrm{N}(\mathrm{x})$ ) minus the number of AVMs that hemorrhaged at earlier ages, $\mathrm{M}(\mathrm{x})$. Thus,

$$
\begin{aligned}
& \mathrm{M}(\mathrm{x})=\operatorname{sum} \mathrm{n}(\mathrm{x}) ; \mathrm{x}=0 \text { to } \mathrm{x}-1 \\
& \text { and } \\
& \mathrm{n}(\mathrm{x})=\mathrm{r} \times(\mathrm{N}(\mathrm{x})-\mathrm{M}(\mathrm{x}))
\end{aligned}
$$

Since $\mathrm{n}(\mathrm{x})$ is known from our data, and thereby also $\mathrm{M}(\mathrm{x})$, we can now use the requirement of constant level of $\mathrm{N}(\mathrm{x})$ at adult ages to determine the value of the age-independent annual risk, with the following simple procedure:

1) set an assumed value for the risk $r$

2) use the relation [eq. 2] to obtain $\mathrm{N}(\mathrm{x})$.

If the curve $N(x)$ is flat for adult ages, $x$, we have guessed the correct value. Otherwise, we repeat steps 1 and 2 until we obtain a curve $\mathrm{N}(\mathrm{x})$ that exhibits a constant level at adult ages. $\mathrm{N}\left(\mathrm{x}_{\max }\right)$ should be equal to the total number of patients in the observed population.

\section{Results}

\section{Universal Shape of the Age Distribution Curve}

The "old" and "new" patient data for the age distribution of the first hemorrhage are compared in Fig. 1. The age distributions were found to be similar for the 2 data sets as well as when comparing patients initially treated with GKS to those initially treated with other methods. The agreement between the curves illustrates the universality of the shape of the age distribution and its independence of the specifics of the patient population. Another support for the universality of the curve is that a similar age distribution was reported earlier. ${ }^{5}$

Comparing the age distributions between male and female patients (Fig. 2), a significant difference was found, with a substantially higher relative incidence of hemorrhage for females between 15 and 35 years old (see below). This represents a greater risk for hemorrhage for females in their fertile years. An age-independent annual risk for hemorrhage is hence not compatible with the age distribu-

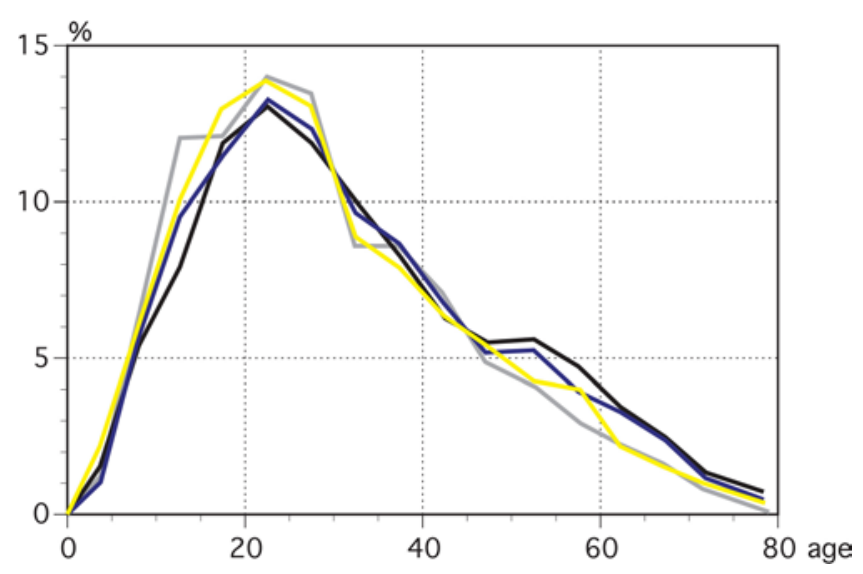

FIG. 1. Age distributions in years presented as the percentage of the total number of hemorrhages (per 5-year interval) for "old" (KLJS; gray curve) and "new" (patients added to KLJS; black curve) observations. The blue curve represents patients initially managed with GKS and the yellow curve the other patients.

tion for females. We therefore primarily used the age distribution for male patients when determining the annual risk for AVM hemorrhage (Fig. 3).

\section{Annual Risk for Hemorrhage}

The curves in Fig. 4 represent assumed values of the annual risk for hemorrhage of $2 \%, 3 \%$, and $4 \%$. We see that a value close to $3 \%$ is needed in order to fulfill the requirement of a constant level of $\mathrm{N}$ at adult age. By using linear regression for the age interval 25-60 years, the best fit was found to be an annual risk of 3.1\% (Fig. 5). It can be concluded that a reasonable estimate of the uncertainty in the determined risk is less than $\pm 0.2 \%$.

Note that this average annual risk means that, on average, about two-thirds of all patients with an unruptured AVM at the age of 25 years will have experienced a hemorrhage before the age of 60 years. Conversely, about one-

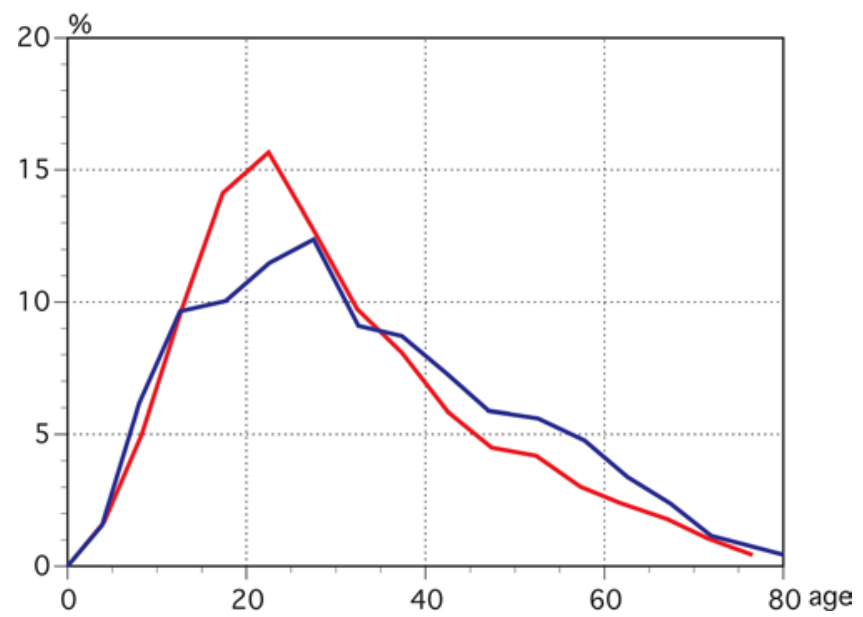

FIG. 2. Age distributions presented as the percentage of the total number of hemorrhages (per 5-year interval) for men (blue curve) and women (red curve). There were 1803 men and 1722 women in the population. 


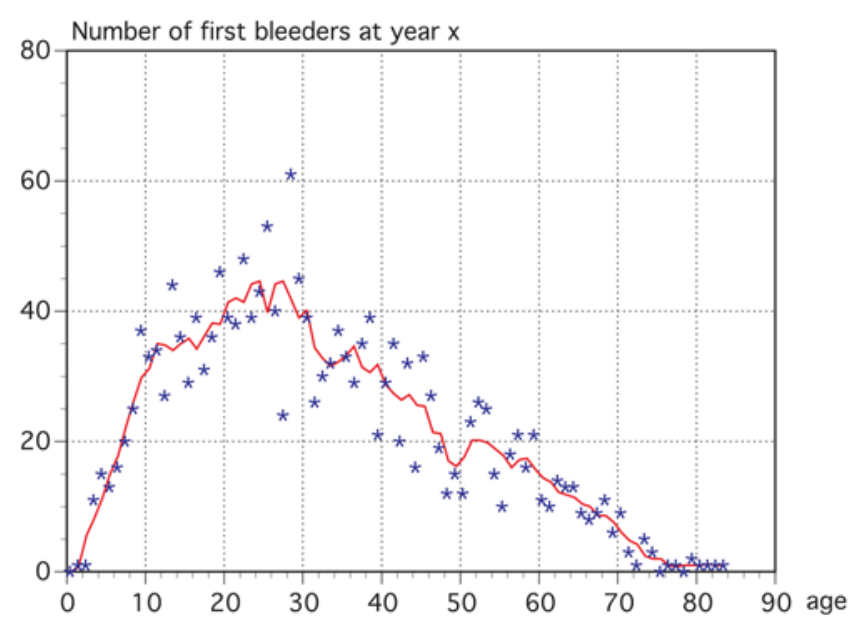

FIG. 3. Age distribution $n(x)$ of the first hemorrhage in the male population. The stars represent the annual values and the curve the 5-year moving average.

third of the same population will reach the age of 60 years without AVM hemorrhage, despite the annual risk of $3.1 \%$.

So, how can we determine the risk for hemorrhage without studying patients with unruptured AVMs? The answer is that the peak and subsequent slope of the hemorrhage age distribution curve (Fig. 1) is directly related to the magnitude of the risk for hemorrhage. The higher the risk, the fewer unruptured AVMs that will remain at older ages. Hence, for greater risk, the age distribution curve will exhibit a higher peak and a steeper slope.

As expected (see Methods), the constant level of $\mathrm{n}(\mathrm{x})$ at adult ages 25-60 years is higher than the total number $\left(\mathrm{N}\left(\mathrm{x}_{\max }\right)=1803\right)$ of observed AVMs that hemorrhaged in the male population studied.

\section{Effect of AVM Parameters on the Risk for Hemorrhage}

Our study is based on the assumption of an age-independent annual risk for AVM hemorrhage. As shown

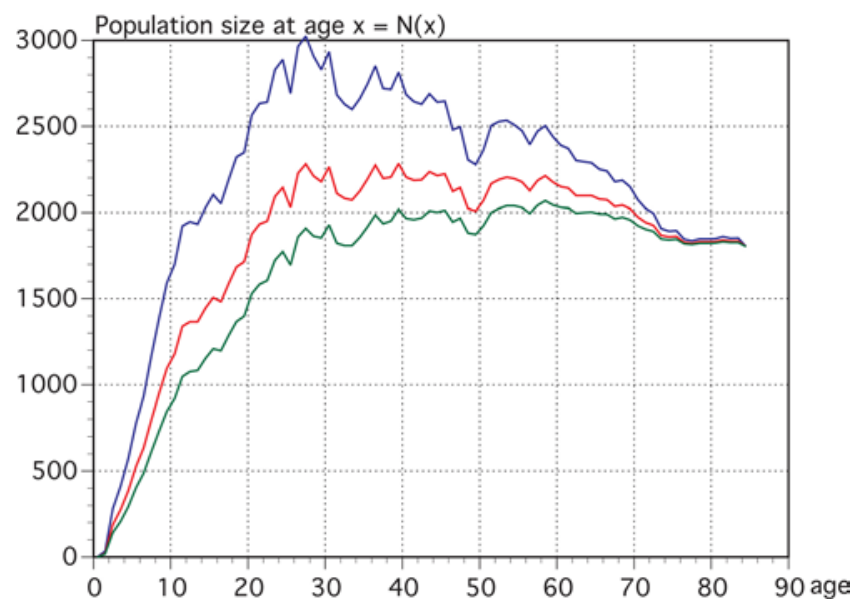

FIG. 4. The (virtual) population size $\mathrm{N}$ as function of age $(\mathrm{x})$ for 3 different assumed values of the risk: $2 \%$ (blue), $3 \%(r e d)$, and $4 \%$ (green). Only the male part of the population is analyzed here.

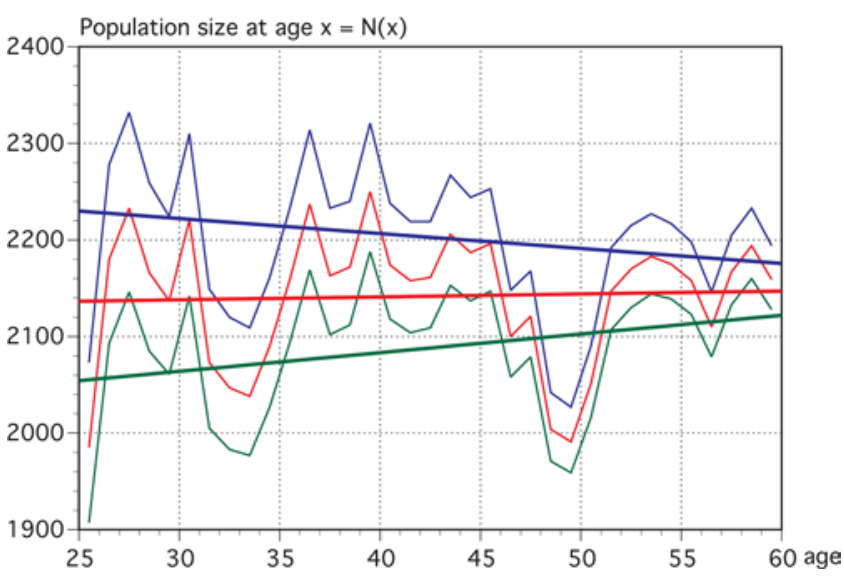

FIG. 5. Closeup of the linear regression for $N(x)$ at ages 25-60 years for 3 different assumptions for the risk $r$. The thin curves (blue $2.9 \%$, red $3.1 \%$, and green $3.3 \%$ ) represent the 5 -year moving average values and the straight lines the corresponding linear curve fits.

above, this is not correct for the female patient population. When dividing the male patient population into subgroups, the low number of patients gives a substantially higher uncertainty in the risk determination. However, the possible influence of AVM volume and location should be sex independent. We can therefore use information from both male and female patients in order to get a larger population and thereby a lower uncertainty. The risk found will be somewhat too high, but the relation between the risks for different groups should be accurate. To assess the magnitude of the error introduced by analyzing the entire patient population, we note that the (flawed) annual risk that results when using data from all patients in the method described above is $4 \%$. This finding is compatible with the findings of Ondra et al. ${ }^{25}$

\section{Factors Affecting the Risk for Hemorrhage}

As seen in Fig. 4, $\mathrm{N}(\mathrm{x})$ increases up to 25 years and decreases after 60 years of age. As a consequence, we decided to use linear regressions for this age interval for the following analyses.

\section{AVM Volume and the Risk for Hemorrhage}

The AVM population was divided in 2 different ways. First, the AVMs were divided into 2 groups: AVM volumes of $\leq 2 \mathrm{~cm}^{3}$ or $>2 \mathrm{~cm}^{3}$. The subgroup of AVMs $\geq 10$ $\mathrm{cm}^{3}$ was analyzed separately. The linear regression with a $3 \%$ estimated annual risk for the male population fits well in all categories, i.e., results in horizontal lines between 26 and 60 years of age. The same was found when using $4 \%$ for the total patient population. This suggests that the impact of AVM volume on the risk for hemorrhage is small.

\section{AVM Location and the Risk for Hemorrhage}

The best fits with a horizontal linear regression for males were found for a $3 \%$ risk for peripherally located AVMs, a $4 \%$ risk for central ones, and a $1 \%$ risk for cerebellar AVMs. The corresponding rates were $3 \%, 5 \%$, and 
$2 \%$, respectively, for the entire patient population. This indicates that centrally located AVMs carry an approximately $1 \%$ higher annual risk than peripheral ones. This result was confirmed by studying a subset of 2565 patients (1429 with peripheral and 1136 with central AVMs) who underwent GKS. The cumulative 2-year post-GKS hemorrhage rate was $3.6 \%$ among patients with peripheral AVMs and $4.6 \%$ among patients with central AVMs (p $=0.02$ ).

The risk for hemorrhage for infratentorial AVMs seems to be lower than that for supratentorial ones, but the uncertainty is high due to the small number of cerebellar AVMs (345).

\section{Assessment of the Risk for Hemorrhage in the Female Patient Population}

As seen in Fig. 2, relatively more females than males have their first hemorrhage between 15 and 35 years of age. With $\mathrm{N}(\mathrm{x})$ known for the male part of the population, we can use this to derive the variation of the annual risk for women that would result in the same shape of the population development as for men. This analysis shows that females have a temporarily increased risk for hemorrhage around between 15 and 35 years of age. Whether this is due to pregnancy or hormonal factors can be tested by comparing the incidence of hemorrhages among males and nonpregnant females in this age range with patent AVMs.

For this purpose, we studied the subgroup of patients who were between 15 and 35 years at the time of GKS. These patients have a persisting risk for hemorrhage between GKS and obliteration, normally occurring 2 years or more after the treatment, during which time women were advised to avoid pregnancy.

Follow-up data for this age group for the first 2 years following GKS were therefore collected, and the dates of hemorrhages were registered. There were 629 males and 708 females with a mean follow-up time of 1.8 years. The data were analyzed in a Kaplan-Meier plot and the resulting cumulative hemorrhage rates showed a slightly but insignificantly lower incidence for females than for males $(\mathrm{p}=0.73)$. This is in sharp contrast to the behavior of the curves in Fig. 2, where females have a large overrepresentation of hemorrhages in this age range, thus indicating a substantially higher risk for females. Hence, we can conclude that the substantially higher risk observed for females in their fertile years can be attributed to pregnancy.

\section{Discussion}

A fundamental assumption for the present analysis is that the risk of hemorrhage is age independent in adult patients. The results from this study support the validity of this assumption for ages up to about 60 years. The assumption may be incorrect for ages above 60 years, where AVM-unrelated deaths affect the results. In addition, an intracerebral hemorrhage in an older patient is less likely to be investigated with angiography and thus AVMs may be underdiagnosed in elderly patients.

One should note that the assumption that AVMs present at birth carry the same risk for hemorrhage as AVMS in adults is incompatible with our data. We cannot conclude whether AVMs are congenital and develop in early years of life or if they are not congenital and develop after birth. The only difference between these 2 scenarios is that the development starts before or after birth. A plausible consequence of our findings is that AVMs found in pediatric patients are likely to be immature compared with AVMs in adults. This was investigated by comparing the hemorrhage rate the first 2 years after GKS in 560 pediatric and 2319 adult patients. The 2-year hemorrhage rate was found to be $2.3 \%$ for pediatric and $4.5 \%$ for adult patients $(p=0.02)$. The consequence of such a lowered risk for the results presented in Fig. 4 would only be a more rapid increase in the AVM population $\mathrm{N}(\mathrm{x})$ development at young ages, while leaving the results for adult ages unchanged.

The $3.1 \%$ risk determined in this study should be interpreted as an average value representative for a large AVM patient population. There are natural variations between different individuals and subgroups, and possibly variation in time for individual patients. However, the average risk for a large patient population will be independent of such specific variations. The observed change in risk following a hemorrhage ${ }^{12,13,31,32}$ is not relevant for this study, as it addresses the risk for the first hemorrhage only.

It has been suggested in the literature that the risk for hemorrhage is highest during the first years after becoming symptomatic and that it decreases continuously thereafter. ${ }^{12,13,31,32}$ A direct consequence of this hypothesis is that the average risk for hemorrhage must decrease with age. This is not compatible with the age distribution in our patient population. More than $20 \%$ of the AVMs would have to develop after the age of 25 years for our data to be compatible with an assumption of a linear decrease in risk from $3 \%$ to $1.5 \%$ between 25 and 60 years. Decreases from $4 \%$ to $2 \%$ and from $2 \%$ to $1 \%$ yielded similar results.

It is highly unlikely that a significant portion of AVMs develop during adulthood. De novo AVMs in patients older than 25 years are so rare that they are published as case reports. ${ }^{3,7,19,21,22,24,29,30}$ Shi et al. and Pabaney et al. suggested that AVM development in adult patients may be "triggered by ischemic and/or traumatic events." 27,29 The number of de novo AVMs in adults is consequently so low that it does not affect the conclusions made in this study. Hence, we must conclude that the assumption of an annual hemorrhage risk that decreases after becoming symptomatic very likely is incorrect.

\section{Conclusions}

The large patient population studied here was shown to give an essentially universal shape of the age distribution for the occurrence of the first AVM hemorrhage. From this age distribution, we could conclude that the average annual risk for hemorrhage in unruptured AVMs in adult patients is around $3.1 \%$ with an uncertainty of less than $\pm 0.2 \%$, without the need for follow-up information. The observed age distribution for AVM hemorrhage is incompatible with AVMs present at birth having the same hemorrhage risk as AVMs in adults. Plausibly, they instead develop in the early years of life, possibly with a lower hemorrhage risk during that time period. 
The risk for hemorrhage was found to be essentially unrelated to AVM volume. However, AVM location affects the risk, being highest for centrally located AVMs. The risk for AVM rupture is significantly increased for females in their fertile years, which was concluded to be due to pregnancy. This conclusion could be reached by complementing the analysis with a follow-up study of males and nonpregnant females over a 2 -year period post-GKS.

\section{Acknowledgments}

We are deeply indebted to the late Ladislau Steiner, MD, PhD, who generously shared his data with B.K. during his work on his thesis.

The work was carried out with internal funding at the respective site. The authors were free of any external influence in the analysis of the data and the writing of the manuscript. The decision to submit the paper for publication was made jointly by the authors. The corresponding author had the final responsibility for submitting the paper for publication.

\section{References}

1. Akimoto H, Komatsu K, Kubota Y: Symptomatic de novo arteriovenous malformation appearing 17 years after the resection of two other arteriovenous malformations in childhood: case report. Neurosurgery 52:228-232, 2003

2. Brown RD Jr, Wiebers DO, Forbes G, O'Fallon WM, Piepgras DG, Marsh WR, et al: The natural history of unruptured intracranial arteriovenous malformations. J Neurosurg 68:352-357, 1988

3. Bulsara KR, Alexander MJ, Villavicencio AT, Graffagnino $\mathrm{C}$ : De novo cerebral arteriovenous malformation: case report. Neurosurgery 50:1137-1141, 2002

4. Can A, Gross BA, Du R: The natural history of cerebral arteriovenous malformations. Handb Clin Neurol 143:15-24, 2017

5. Crawford PM, West CR, Chadwick DW, Shaw MD: Arteriovenous malformations of the brain: natural history in unoperated patients. J Neurol Neurosurg Psychiatry 49:1-10, 1986

6. Eguchi S, Aihara Y, Yamaguchi K, Okada Y: Limitations of fetal ultrasonography and magnetic resonance imaging in prenatal diagnosis of congenital cerebral arteriovenous malformations with hemorrhagic onset. J Neurosurg Pediatr 10:154-158, 2012

7. Friedman JA, Pollock BE, Nichols DA: Development of a cerebral arteriovenous malformation documented in an adult by serial angiography. Case report. J Neurosurg 93:1058-1061, 2000

8. Fults D, Kelly DL Jr: Natural history of arteriovenous malformations of the brain: a clinical study. Neurosurgery 15:658-662, 1984

9. Gabriel EM, Sampson JH, Wilkins RH: Recurrence of a cerebral arteriovenous malformation after surgical excision. Case report. J Neurosurg 84:879-882, 1996

10. Graf CJ, Perret GE, Torner JC: Bleeding from cerebral arteriovenous malformations as part of their natural history. $\mathbf{J}$ Neurosurg 58:331-337, 1983

11. Halim AX, Johnston SC, Singh V, McCulloch CE, Bennett JP, Achrol AS, et al: Longitudinal risk of intracranial hemorrhage in patients with arteriovenous malformation of the brain within a defined population. Stroke 35:1697-1702, 2004

12. Hernesniemi JA, Dashti R, Juvela S, Väärt K, Niemelä M, Laakso A: Natural history of brain arteriovenous malformations: a long-term follow-up study of risk of hemorrhage in 238 patients. Neurosurgery 63:823-831, 2008

13. Itoyama Y, Uemura S, Ushio Y, Kuratsu J, Nonaka N, Wada
$\mathrm{H}$, et al: Natural course of unoperated intracranial arteriovenous malformations: study of 50 cases. J Neurosurg 71:805809, 1989

14. Kader A, Goodrich JT, Sonstein WJ, Stein BM, Carmel PW, Michelsen WJ: Recurrent cerebral arteriovenous malformations after negative postoperative angiograms. J Neurosurg 85:14-18, 1996

15. Karlsson B, Lindquist C, Johansson A, Steiner L: Annual risk for the first hemorrhage from untreated cerebral arteriovenous malformations. Minim Invasive Neurosurg 40:40-46, 1997

16. Kim H, Al-Shahi Salman R, McCulloch CE, Stapf C, Young WL: Untreated brain arteriovenous malformation: patientlevel meta-analysis of hemorrhage predictors. Neurology 83:590-597, 2014

17. Kim H, Sidney S, McCulloch CE, Poon KY, Singh V, Johnston SC, et al: Racial/ethnic differences in longitudinal risk of intracranial hemorrhage in brain arteriovenous malformation patients. Stroke 38:2430-2437, 2007

18. Lasjaunias P: A revised concept of the congenital nature of cerebral arteriovenous malformations. Interv Neuroradiol 3:275-281, 1997

19. Lindqvist M, Karlsson B, Guo WY, Kihlström L, Lippitz B, Yamamoto M: Angiographic long-term follow-up data for arteriovenous malformations previously proven to be obliterated after gamma knife radiosurgery. Neurosurgery 46:803-810, 2000

20. Mast H, Young WL, Koennecke HC, Sciacca RR, Osipov A, Pile-Spellman J, et al: Risk of spontaneous haemorrhage after diagnosis of cerebral arteriovenous malformation. Lancet 350:1065-1068, 1997

21. Miyasaka Y, Nakahara K, Takagi H, Hagiwara H: Development of multiple cerebral arteriovenous malformations documented in an adult by serial angiography. Case report. J Neurosurg 98:190-193, 2003

22. Mizutani T, Tanaka H, Aruga T: Total recanalization of a spontaneously thrombosed arteriovenous malformation. Case report. J Neurosurg 82:506-508, 1995

23. Mohr JP, Parides MK, Stapf C, Moquete E, Moy CS, Overbey JR, et al: Medical management with or without interventional therapy for unruptured brain arteriovenous malformations (ARUBA): a multicentre, non-blinded, randomised trial. Lancet 383:614-621, 2014

24. Morales-Valero SF, Bortolotti C, Sturiale C, Lanzino G: Are parenchymal AVMs congenital lesions? Neurosurg Focus 37(3):E2, 2014 [Erratum in Neurosurg Focus 39(1):E14, 2015]

25. Ondra SL, Troupp H, George ED, Schwab K: The natural history of symptomatic arteriovenous malformations of the brain: a 24-year follow-up assessment. J Neurosurg 73:387391, 1990

26. O'Shaughnessy BA, DiPatri AJ Jr, Parkinson RJ, Batjer HH: Development of a de novo cerebral arteriovenous malformation in a child with sickle cell disease and moyamoya arteriopathy. Case report. J Neurosurg 102 (2 Suppl):238-243, 2005

27. Pabaney AH, Rammo RA, Tahir RA, Seyfried D: Development of de novo arteriovenous malformation following ischemic stroke: case report and review of current literature. World Neurosurg 96:608.e5-608.e12, 2016

28. Petridis AK, Fischer I, Cornelius JF, Kamp MA, Ringel F, Tortora A, et al: Demographic distribution of hospital admissions for brain arteriovenous malformations in Germanyestimation of the natural course with the big-data approach. Acta Neurochir (Wien) 158:791-796, 2016

29. Shi S, Gupta R, Moore JM, Griessenauer CJ, Adeeb N, Motiei-Langroudi R, et al: De novo AVM formation following venous sinus thrombosis and prior AVM resection in adults: report of 2 cases. J Neurosurg 128:506-510, 2018 
30. Shidoh S, Kobayashi M, Akaji K, Kano T, Tanizaki Y, Mihara B: De novo arteriovenous malformation after aneurysm clipping. NMC Case Rep J 4:89-92, 2017

31. Stapf C, Mast H, Sciacca RR, Choi JH, Khaw AV, Connolly ES, et al: Predictors of hemorrhage in patients with untreated brain arteriovenous malformation. Neurology 66:1350-1355, 2006

32. Yamada S, Takagi Y, Nozaki K, Kikuta K, Hashimoto N: Risk factors for subsequent hemorrhage in patients with cerebral arteriovenous malformations. J Neurosurg 107:965972, 2007

\section{Disclosures}

Dr. Söderman: consultant for J\&J and Archer Research; patent holder with Neurvana; and long-term scientific collaboration with Philips Healthcare.

\section{Author Contributions}

Conception and design: Johansson, Karlsson. Acquisition of data: Karlsson, Yang, Jokura, Yamamoto, Kawagishi, Guo, Beute, Pan,
Chung, Söderman, Aiyama, Yeo. Analysis and interpretation of data: Johansson, Karlsson. Drafting the article: Johansson, Karlsson. Critically revising the article: all authors. Reviewed submitted version of manuscript: all authors. Approved the final version of the manuscript on behalf of all authors: Johansson. Statistical analysis: Johansson, Karlsson.

\section{Supplemental Information}

Previous Presentations

Portions of this work were presented at the 19th Leksell Gamma Knife Society Meeting, Dubai, United Arab Emirates, March 4-8, 2018.

\section{Correspondence}

Arne V. Johansson: KTH Royal Institute of Technology, Stockholm, Sweden. viktor@kth.se. 